\title{
Cannabinoid receptor 1 signaling in hepatocytes and stellate cells does not contribute to NAFLD
}

\author{
Simeng Wang, ${ }^{1}$ Qingzhang Zhu, ${ }^{1}$ Guosheng Liang, ${ }^{1,2}$ Tania Franks, ${ }^{3}$ Magalie Boucher, ${ }^{3}$ Kendra K. Bence, ${ }^{4}$ Mingjian Lu, ${ }^{4}$ \\ Carlos M. Castorena, ' Shangang Zhao, ' Joel K. Elmquist, ${ }^{1}$ Philipp E. Scherer, ${ }^{1}$ and Jay D. Horton ${ }^{1,2}$ \\ 'Department of Internal Medicine and 'Department of Molecular Genetics, University of Texas Southwestern Medical Center, Dallas, Texas, USA. ${ }^{3}$ Drug Safety Research and Development, Pfizer Inc., Groton, \\ Connecticut and Cambridge, Massachusetts, USA. ${ }^{4}$ Internal Medicine Research Unit, Pfizer Worldwide Research, Development, and Medical, Cambridge, Massachusetts, USA.
}

\begin{abstract}
The endocannabinoid system regulates appetite and energy expenditure and inhibitors of cannabinoid receptor 1 (CB-1) induce weight loss with improvement in components of the metabolic syndrome. While CB-1 blockage in brain is responsible for weight loss, many of the metabolic benefits associated with CB-1 blockade have been attributed to inhibition of CB-1 signaling in the periphery. As a result, there has been interest in developing a peripherally restricted CB-1 inhibitor for the treatment of nonalcoholic fatty liver disease (NAFLD) that would lack the unwanted centrally mediated side effects. Here, we produced mice that lacked CB-1 in hepatocytes or stellate cells to determine if CB-1 signaling contributes to the development of NAFLD or liver fibrosis. Deletion of CB-1 in hepatocytes did not alter the development of NAFLD in mice fed a high-sucrose diet (HSD) or a high-fat diet (HFD). Similarly, deletion of CB-1 specifically in stellate cells also did not prevent the development of NAFLD in mice fed the HFD, nor did it protect mice from carbon tetrachloride-induced fibrosis. Combined, these studies do not support a direct role for hepatocyte or stellate cell CB-1 signaling in the development of NAFLD or liver fibrosis.
\end{abstract}

\section{Introduction}

Nonalcoholic fatty liver disease (NAFLD) is the most common condition affecting the liver in the United States, owing to its association with obesity and the metabolic syndrome (1). The largest study to date using magnetic resonance spectroscopy to quantify liver triglyceride (TG) content showed that approximately $33 \%$ of individuals have hepatic steatosis (2). NAFLD encompasses a continuum of histological findings that starts with steatosis that can progress to nonalcoholic steatohepatitis (NASH), which is characterized by inflammation and cell death, and eventually cirrhosis (3). Given the large number of individuals afflicted with this condition, there is a clear need to develop effective and safe therapies to treat NAFLD.

Over the past 2 decades, significant progress has been made in defining how and why fat accumulates in the liver. NAFLD develops as a result of insulin resistance, which is most often associated with obesity $(4,5)$. Recent studies suggest that up to $38 \%$ of the fatty acids in TGs in liver are derived from de novo lipogenesis, while approximately $15 \%$ come from diet and the remaining from free fatty acids in the blood (6). The genes required for de novo lipogenesis are all regulated by the transcription factor sterol regulatory element-binding protein-1c (SREBP-1c), which is activated by insulin and is induced in animal models and humans with fatty liver $(7$, 8 ), and carbohydrate response element-binding protein (ChREBP), which is activated by glucose $(9,10)$. Blocking the lipogenic path-

Conflict of interest: JDH is a consultant for Pfizer. JKE and GL conduct sponsored research with Pfizer.

Copyright: () 2021, American Society for Clinical Investigation.

Submitted: June 11, 2021; Accepted: August 31, 2021; Published: November 15, 2021

Reference information: / Clin Invest. 2021;131(22):e152242.

https://doi.org/10.1172/JCl152242. way through the inhibition of SREBPs, acetyl-CoA carboxylase (the first committed enzyme in the fatty acid synthesis pathway), or diacylglycerol acyltransferase 2 (the enzyme that catalyzes the final step in TG synthesis), markedly reduces hepatic steatosis in animal models of NAFLD (11-13) and in humans $(11,12,14,15)$.

A second way to reduce liver lipogenesis is to improve insulin resistance through weight loss (1). To date, weight loss either through dietary restriction, bariatric surgery, or possibly GLP-1 agonism has been the most reliable intervention to consistently improve NAFLD histologically (16-19). Based on these studies, it is likely that a therapeutic agent that induces an $8 \%$ to $10 \%$ weight loss would likely have a positive impact on NAFLD progression.

Cannabinoid receptor 1 (CB-1) has a well-defined role in regulating appetite and energy expenditure through its actions in the brain $(20,21)$. Administration of rimonabant, a centrally acting inverse agonist of CB-1, resulted in significant weight loss and improved measures of the metabolic syndrome in humans (22, 23). Unfortunately, the drug had to be withdrawn from the market due to central side effects and before its efficacy in NAFLD was fully assessed (24). More recent investigations have suggested that the beneficial effects of CB-1 antagonism on NAFLD is mediated through peripheral CB-1 proteins (25). Mice that lacked CB-1 in hepatocytes and fed an HFD had similar caloric intake and weight gain to those of wild-type mice but had lower hepatic TGs, reduced measures of insulin resistance, and lower rates of hepatic lipogenesis $(26,27)$. It was suggested that signaling through CB-1 led to increased SREBP-1c levels and increased hepatic lipogenesis. Liu et al. (28) reported that CB-1 in liver was necessary and sufficient for the development of diet-induced hepatic insulin resistance and that CB-1 signaling in hepatocytes improved glycemic control via increased energy expenditure (29). If true, it is possible that a peripherally restricted antagonist of $\mathrm{CB}-1$ could be developed that 
would lack the deleterious neuropsychiatric side effects but still be effective for the treatment of NAFLD (30).

To further interrogate mechanisms by which CB-1 signaling might regulate lipogenesis and the development of NAFLD, we independently generated mice that lack CB-1 in hepatocytes or hepatic stellate cells (HSCs). Here, we show that the deletion of the CB-1 gene (Cnrl) did not prevent the development of NAFLD in mice fed an HSD or HFD. Similarly, the deletion of Cnr1 specifically in HSCs did not alter the severity of NAFLD in response to an HFD nor did it protect mice from carbon tetrachloride-induced $\left(\mathrm{CCl}_{4}\right.$-induced) fibrosis.

\section{Results}

Metabolic consequences of Cnr1 deletion in hepatocytes. Conditional Cnr1-knockout mice ( $\left.\mathrm{Cnr}^{1 / f / f}\right)$ were generated using CRISPR/Cas9 genome editing to insert one loxP site upstream and another loxP site downstream of exon 2 of the Cnr1 allele (Supplemental Figure 1A; supplemental material available online with this article; https:// doi.org/10.1172/JCI152242DS1). $C n r 1^{f l / f l}$ mice were bred with mice expressing Cre recombinase under the control of the mouse zona pellucida 3 promoter (ZP3-Cre) (31) to generate a germline deletion in $\mathrm{Cnr} 1\left(\mathrm{Cnr}^{\Delta / \Delta}\right)$ mice. $\mathrm{Cnr1}$ mRNA was undetectable in multiple tissues of $C n r 1^{\Delta / \Delta}$ mice, which confirmed that the loxP sites were functional and that Cre recombinase successfully knocked out CB-1 expression (32). We further assessed the brain, which is enriched in CB-1 expression (33). $\mathrm{Cnr}^{f / / f l}$ mice were crossed with SF1-Cre mice (34) to delete $\mathrm{Cnr} 1$ from SF1-expressing neurons $\left(\mathrm{Cnrl}^{\text {SF1-KO}}\right)$. RNA in situ hybridization showed a significant reduction in Cnr1 mRNA levels in the ventromedial hypothalamus $(\mathrm{VMH})$ of $\mathrm{Cnr1}^{\text {SFI-KO }}$ mice compared with those in $\mathrm{Cnr}^{\mathrm{fl} / \mathrm{fl}}$ mice (32).

The $C n r 1^{f / f l}$ mice (Supplemental Figure 1B) were next bred with transgenic mice expressing Cre recombinase under the control of the hepatocyte-specific albumin promotor (Alb-Cre) to produce the hepatocyte-specific Cnrl-knockout mice (Hep-Cnr1--)). Real-time qPCR was used to quantify the expression of Cnr1

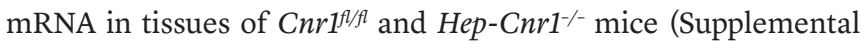
Figure 1C). Cnr1 expression in whole-liver RNA extracts was very low compared with the expression in the hypothalamus, white adipose tissue, brown adipose tissue, and muscle.

Inasmuch as previous studies have suggested that caloric excess upregulates CB-1 expression and induces SREBP-1c in hepatocytes (27), we first investigated whether hepatic Cnr1 mRNA levels changed in livers of mice fed an HSD or HFD. HSD feeding for 17 weeks resulted in obesity, hepatocyte damage, and liver steatosis as evidenced by a marked increase in body weight, plasma alanine aminotransferase (ALT) and aspartate aminotransferase (AST) levels, liver histology, and liver TG and cholesterol levels in wild-type mice (Figure 1, A-C and F, and Figure 2, A and B). No significant differences in response to the HSD were observed between control and $\mathrm{Hep}-\mathrm{Cnrl}^{-/-}$mice (Figure 1). Cnr1 mRNA levels were not induced by the HSD in control livers (Figure 1D); however, the expected induction of SREBP-1 protein as well as a significant increase in the level of mRNAs encoding genes involved in lipogenesis was observed (Figure 2, C-E). Concurrently, 17-week HSD feeding resulted in elevated mRNA levels of genes associated with fibrosis such as collagen type $1 \alpha 1$ (Col1a1) and $\alpha$-smooth muscle actin $(\alpha S M A)$, but there was no evidence of fibrosis as determined by picrosirius red (PSR) staining of liver sections and collagen quantification (Figure 1, E and F). Taken together, these data suggest that CB-1 expression is extremely low in liver and that $C n r 1$ deletion in hepatocytes did not prevent the hepatocellular metabolic dysfunction induced by the HSD.

We next determined whether CB-1 in hepatocytes has a role in regulating glucose metabolism and insulin sensitivity. Hep-Cnr1 ${ }^{-1-}$ and control mice were fed chow or the HSD for 16 weeks. In both cohorts of mice, the HSD led to similarly elevated fasting blood glucose levels and there was no difference in glucose tolerance (Figure 3 , A and B) or insulin sensitivity (Figure 3, C and D). To test whether hepatocyte $\mathrm{Cnr} 1$ deletion altered insulin resistance, $\mathrm{Hep}-\mathrm{Cnr1^{-/ }}$ and control mice were euthanized after a 17-week challenge with the HSD. Hepatocyte Cnr1 deletion did not alter plasma glucose and insulin levels (Figure 3, E and F). These studies demonstrated that hepatocyte-specific deletion of $\mathrm{Cnrl}$ did not prevent glucose intolerance and insulin resistance induced by the HSD.

To determine whether hepatocyte-specific deletion of Cnrl could alter the pathologic features of HFD-induced hepatic steatosis, Hep-Cnr1--and control mice were fed chow or an HFD for 12 weeks before euthanasia. Cnr1 deletion did not alter body weights (Supplemental Figure 2, A and B), plasma ALT and AST levels (Supplemental Figure 2C), glucose tolerance (Supplemental Figure 3, A and B), insulin sensitivity (Supplemental Figure 3, $\mathrm{C}$ and D), liver TG and cholesterol concentrations (Supplemental Figure 3, E and F), or liver histology (Supplemental Figure 2F). There were also no discernable differences in Cnr1 mRNA levels in response to the HFD between control and Hep-Cnr1-/- mice, but similar induction of mRNAs encoding genes involved in fibrogenesis and lipogenesis was observed (Supplemental Figure 2D and Supplemental Figure 3G). PSR staining of liver sections and collagen quantification did not reveal evidence of significant fibrosis induced by HFD (Supplemental Figure 2, E and F). These studies demonstrated that deletion of $\mathrm{Cnr} 1$ does not change hepatic steatosis or the metabolic disorders induced by an HFD.

To determine whether hepatocyte-specific deletion of Cnr1 could reverse the pathologic features of diet-induced hepatic steatosis, $C n r 1^{f l / f l}$ mice were fed an HFD for 8 weeks and then injected with an adeno-associated virus expressing Cre recombinase (AAV-Cre) to delete Cnr1 in hepatocytes or a control (AAV-GFP) virus. Mice were maintained on the HFD for an additional 8 weeks before euthanasia. Cnr1 deletion did not alter body weights (Supplemental Figure 4A), plasma ALT and AST levels (Supplemental Figure 4B), glucose tolerance (Supplemental Figure 4C), insulin sensitivity (Supplemental Figure 4D), or liver TG and cholesterol concentrations (Supplemental Figure 5, A and B). There were also no significant differences in liver histology (Supplemental Figure 5D). These studies showed that an inducible deletion of Cnr1 does not reverse hepatic steatosis or alter the HFD-induced pathological features.

Single-cell RNA sequencing of hepatocytes and HSCs reveals low Cnr1 expression in livers of mice fed chow or HSD. To determine if a small population of cells in the liver express CB-1 at high levels before and/or after HSD feeding, we performed single-cell RNA-sequencing (scRNA-seq) of livers from wild-type mice fed chow or an HSD for 17 weeks and analyzed Cnr1 expression. Previous studies showed that hepatocytes are sensitive to flow sort- 

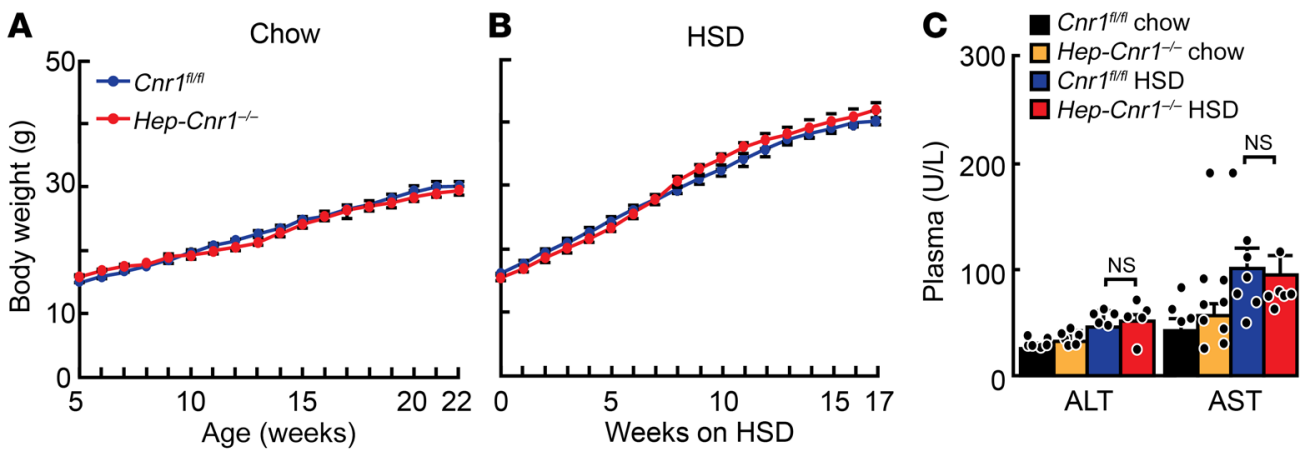

D
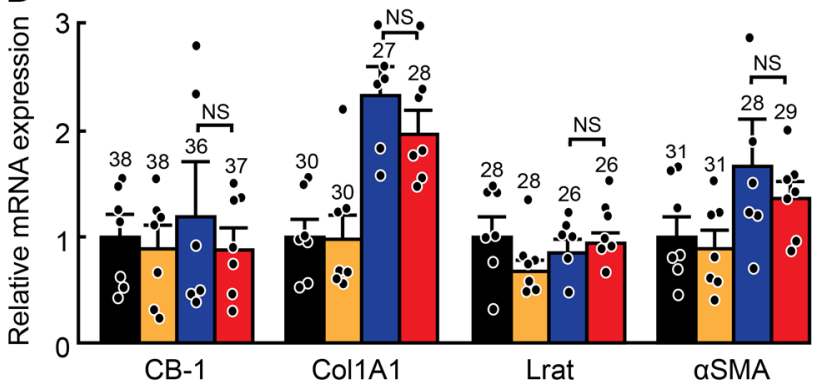

$\mathbf{E}$
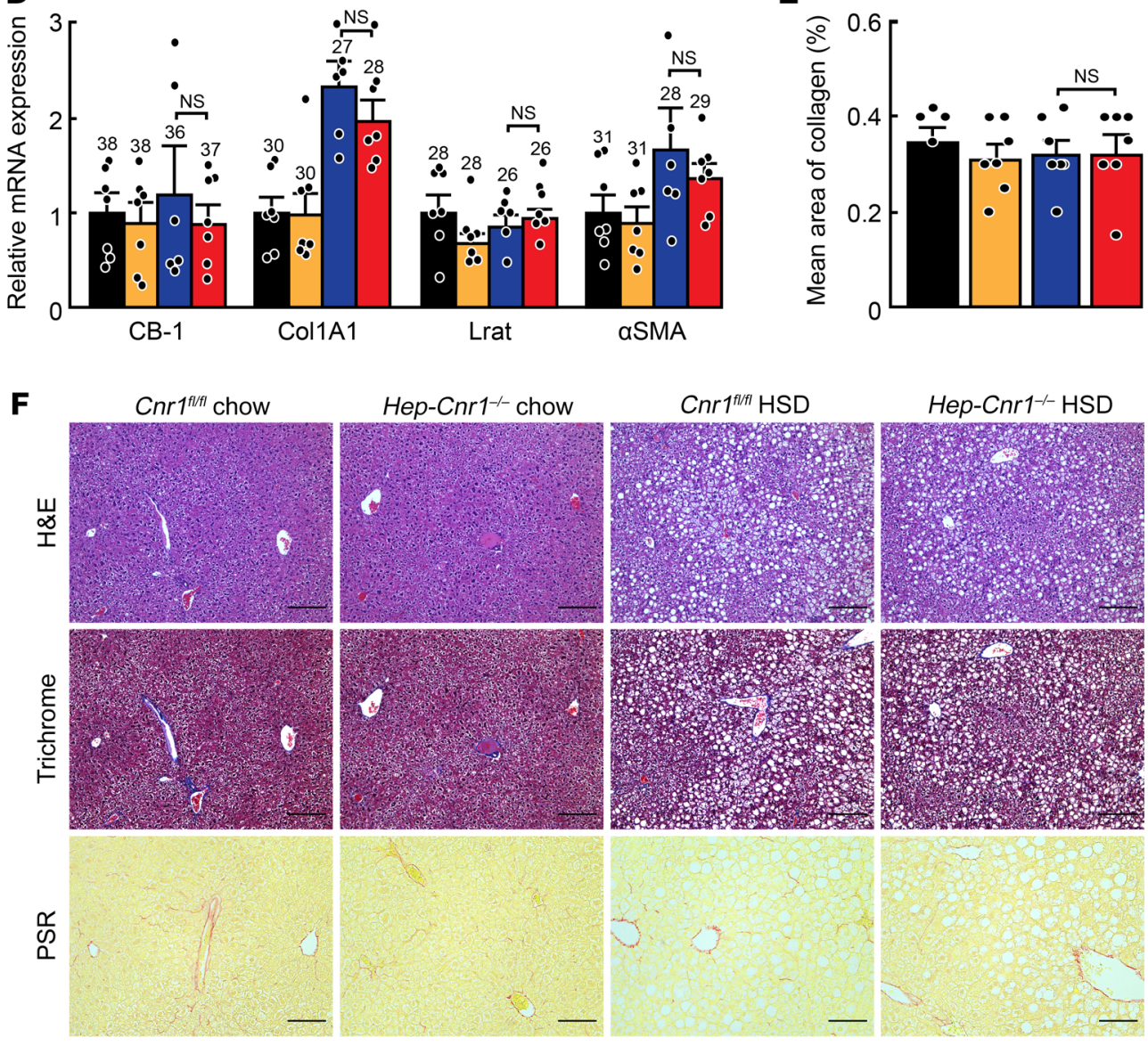

Figure 1. Cnr1 deletion in hepatocytes does not affect body weight, liver function, or diet-induced obesity in 22-week-old mice fed chow or an HSD. Growth curves of chowfed (A) and HSD-fed (B) Cnr ffl/fl

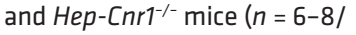
group). Body weights were monitored weekly starting at 5 weeks of age. (C) Plasma ALT and AST levels. (D) Whole-liver RNA was extracted for measurement of relative mRNA levels of Cnr1, Col1a1, Lrat, and Acta2 ( $\alpha$ SMA) quantified by qPCR. $A p o B$ was used as an invariant control. Values are expressed relative to chow-fed $C n r^{f l / f l}$ mice, which was arbitrarily set to 1 . Corresponding mean $C_{t}$ values are denoted above. (E) Mean area of collagen was obtained by calculating the PSRstained red area in the image under a split green channel, as described in the Methods. Results shown as mean \pm SEM, assessed by ANOVA. (F) H\&E, trichrome, and PSR staining of liver sections. Scale bar: $100 \mu \mathrm{m}$. All experiments (A-F) were repeated with a separate cohort of mice and with similar results. ing (35); therefore, we optimized a hepatocyte isolation protocol without flow cytometry to harvest the greatest number of viable hepatocytes for sequencing. A total of 32,020 cell transcriptomes were obtained from 2 pairs of mice (21,891 chow and 10,129 HSD). The single-cell transcriptomes of each group were aggregated and analyzed with the Seurat R pipeline $(36,37)$. Hepatocytes had a higher ratio of mitochondrial genome transcripts than other resident liver cells, likely due to lysis during isolation (35). Nevertheless, our approach resulted in significantly more viable hepatocytes than previously published methods (Supplemental Figure 6, A and B). A total of 17,530 cells were plotted in the chow-fed group (Figure 4A) and 8,256 cells in the HSD-fed group (Figure 4B) with a uniform manifold approximation and projection (UMAP) (38).

A panel of genes was used to confirm the identity of each cluster based on previously published cell type-specific transcrip- tomes $(35,39,40)$. Hepatocyte clusters revealed an abundant expression of $A l b$ (data not shown), which could be further divided into groups of spatially heterogeneous cells based on zonated gene expression profiles along the portal-central axis of the liver lobule using the signature genes cytochrome $\mathrm{P} 450$ family 2 subfamily $\mathrm{F}$ member 2 (Cyp2f2) (periportal region), cytochrome P450 family 2 subfamily E member 1 (Cyp2e1), and cytochrome P450 family 1 subfamily A member 2 (Cyp1a2) (midzonal/pericentral region; Figure 4, C and D, and refs. 41, 42). Collagen type $3 \alpha 1$ (Col3a1) (data not shown) marked the HSC cluster, which can be characterized into 2 general subpopulations of quiescent and activated HSCs. Lecithin retinol acyltransferase (Lrat) was highly expressed in quiescent HSCs, whereas Col1a1 enrichment represented a more activated state of HSCs in both groups (Figure 4, C and D, and ref. 43). HSD feeding led to HSC activation confirmed by an induc- 
A

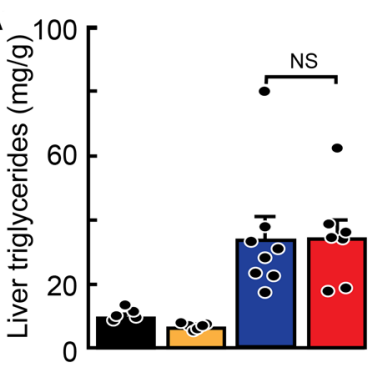

C

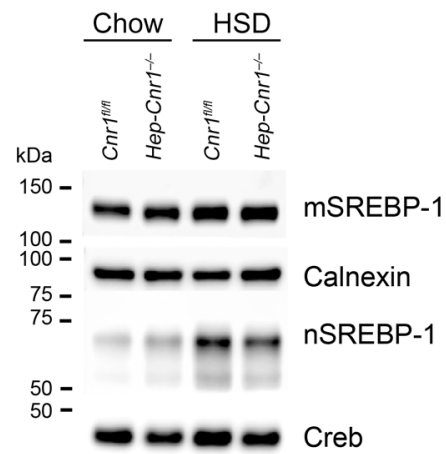

B

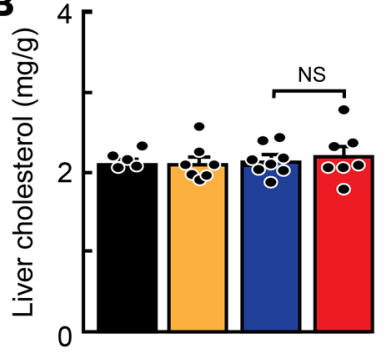

D

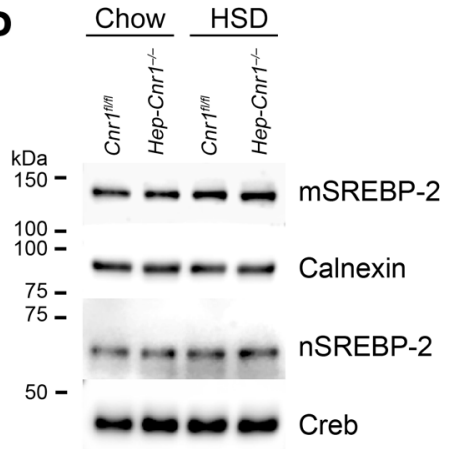

Figure 2. Cnr1 deletion in hepatocytes does not affect liver steatosis in mice fed chow or HSD. The mice used are those described in Figure 1. (A and B) Liver TG and cholesterol levels

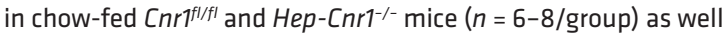
as HSD-fed $\mathrm{Cnr}^{\mathrm{fl} / \mathrm{fl} \text { and } \mathrm{Hep} \text {-Cnr1 }}{ }^{-/-}$mice ( $n=6$-8/group) were measured before euthanasia at 22 weeks of age. (C and $\mathbf{D})$ Membrane and nuclear fractions of SREBP-1 and SREBP-2 expression in pooled liver protein of chow-fed $\mathrm{Cnr}^{\mathrm{fl} / \mathrm{fl} l}$ and $\mathrm{Hep}-\mathrm{Cnr1}^{-1-}$ mice ( $n=6-8$ /group) as well as HSD-fed $\mathrm{Cnr}^{f / / f l}$ and $\mathrm{Hep}-\mathrm{Cnrr}^{-/-}$mice ( $n=6-8 /$ group) euthanized at 22 weeks of age. Calnexin and Creb served as controls for membrane and nuclear proteins, respectively. (E) Total RNA was extracted from each mouse liver, and the relative mRNA expression levels of Srebp-1c, Srebp-2, Chrebp, Acly, Acc1, Fasn, Scd1, and Elovl6 were quantified by real-time PCR. ApoB was used as an invariant control. The values were expressed relative to that of chow-fed $C n r^{f / / f l}$ mice, which was arbitrarily set to 1 . Corresponding mean $C_{t}$ values are denoted above. Results shown as mean \pm SEM, assessed by ANOVA. mSREBP-1, membrane-bound SREBP-1; nSREBP-1, nuclear form of SREBP-1; mSREBP-2, membrane-bound SREBP-2; nSREBP-2, nuclear form of SREBP-2; Acly, ATP-citrate lyase; Chrebp, carbohydrate response element-binding protein; Elovl6, elongation of long chain fatty acids family member 6 ; Fasn, fatty acid synthase; Scd1, stearoyl CoA desaturase 1.

$\mathbf{E}$

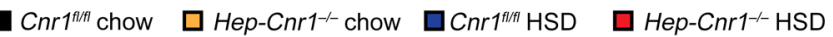

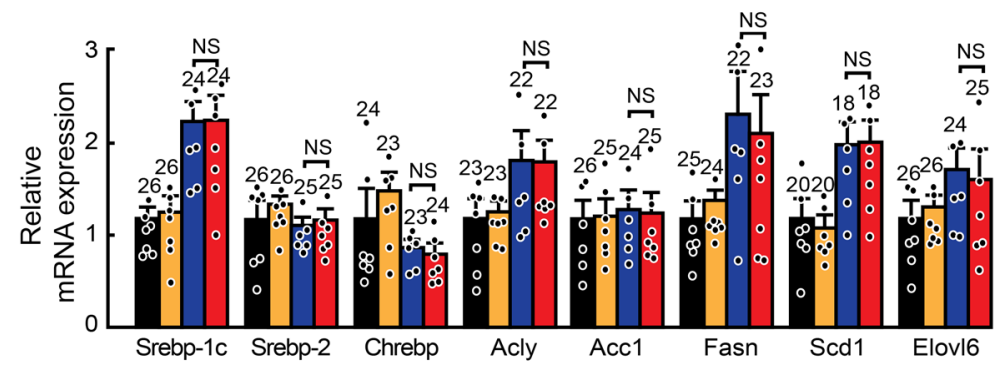

tion of $\alpha S M A$ mRNA expression in HSCs compared with chow-fed mice (Figure 4D). Nonparenchymal liver cells like cholangiocytes, endothelial cells, Kupffer cells, and mixed immune cell types (data not shown) were identified using keratin 19 (Krt19), kinase insert domain receptor $(K d r)$, adhesion $\mathrm{G}$ protein-coupled receptor E1 (Adgre1), and protein tyrosine phosphatase receptor type C (Ptprc), respectively (Supplemental Figure 6, C and D). Next, we queried whether CB-1 is differentially distributed or enriched in a particular cell type. Clustering analysis displayed rare $\mathrm{Cnr} 1$ expression in hepatocytes and HSCs, as well as other nonparenchymal liver cells of mice fed chow or the HSD (Figure 4, C and D). Taken together, the transcriptomes of hepatocytes and HSCs in mice fed chow or HSD verifies that Cnrl expression is extremely low in hepatocytes and HSCs, and its expression was not induced in a subpopulation of cells in the liver by the HSD.

Crn1 deletion in HSCs. Previous studies have suggested that CB-1 expression was induced in cirrhotic livers of humans, and that $\mathrm{CB}-1$ antagonists suppress the progression of liver fibrosis in mice (44). To determine if CB-1 expression in HSCs contributed to the development of fibrosis, we generated mice that lacked CB-1 in HSCs by breeding $C n r 1^{f / f l}$ mice with mice expressing Cre recombinase under the control of a tetracycline-responsive promoter element (TRE-Cre). Offspring with both targeted alleles and the Cre transgene were bred with mice expressing reverse tetracycline-controlled transactivator protein (rtTA) driven by the Lrat (45) promoter. Cnr $1^{f / f l}$ mice with Cre and rtTA were fed a doxycycline diet for 4 weeks to obtain mice with HSC-specific Cnr1 deletion (Hsc-Cnr1 ${ }^{-1}$; Supplemental Figure 7A).

Quiescent HSCs are characterized by the storage of retinyl esters in their cytoplasm (46). Liver injury and in vitro culture drive HSCs to undergo a transdifferentiation to an activated myofibroblast-like cell type, with the distinct feature of losing vitamin A storage (43). To determine if CB-1 was induced during stellate cell activation, HSCs were isolated from wild-type mice and cultured. As previously reported (43), HSCs gradually lost their endogenous retinoid fluorescence and morphologically became myofibroblast-like cells during 6 days in culture (Figure 5A). HSCs cultured for 6 days displayed increased expression of genes associated with activation such as $\alpha S M A$ and Colla1 as well as decreased expression of Lrat, a quiescent HSC-enriched marker (Figure 5B and ref. 45). Cnr1 mRNA levels were present at low levels following HSC isolation and did not change as HSCs became activated (Figure 5B).

To investigate a potential role of stellate cell CB-1 signaling in fibrosis, $\mathrm{CCl}_{4}$ was administered to wild-type and $\mathrm{Hsc}_{-} \mathrm{Cnrl}^{-/-}$mice to induce fibrosis. Chronic exposure of $\mathrm{Hsc}_{-} \mathrm{Cnrl}^{-/-}$and control mice to high-dose $\mathrm{CCl}_{4}$ for 10 weeks via peritoneal administration resulted in extensive liver nodules without significant changes in plasma ALT and AST levels (Figure 5, C and E). $\mathrm{CCl}_{4}$ administra- 

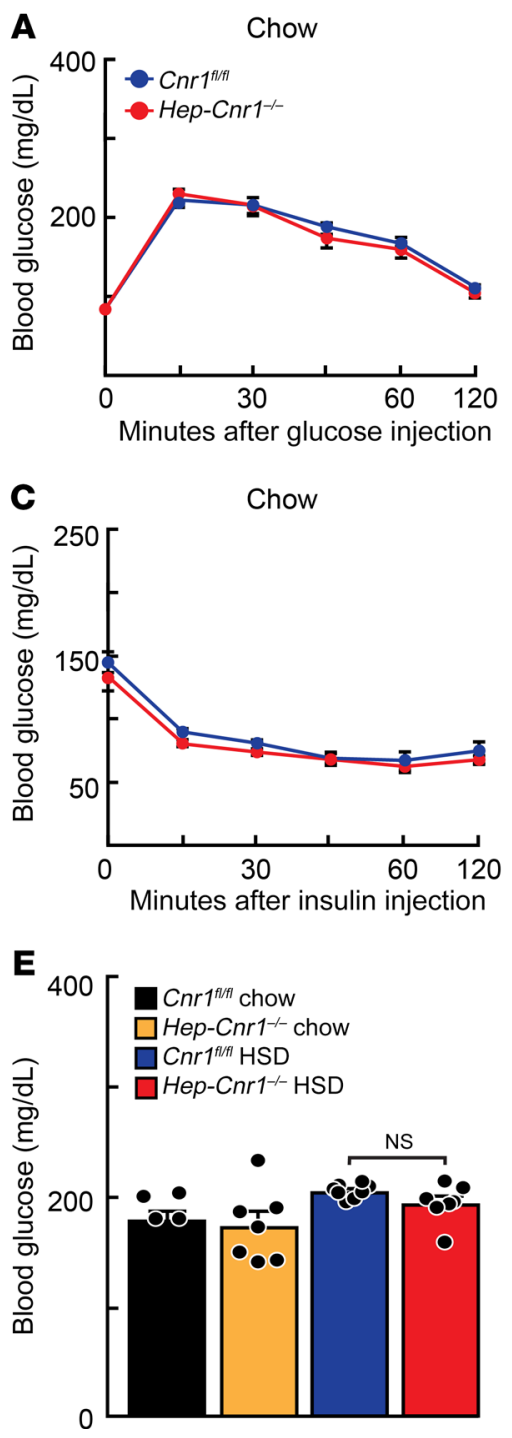

B
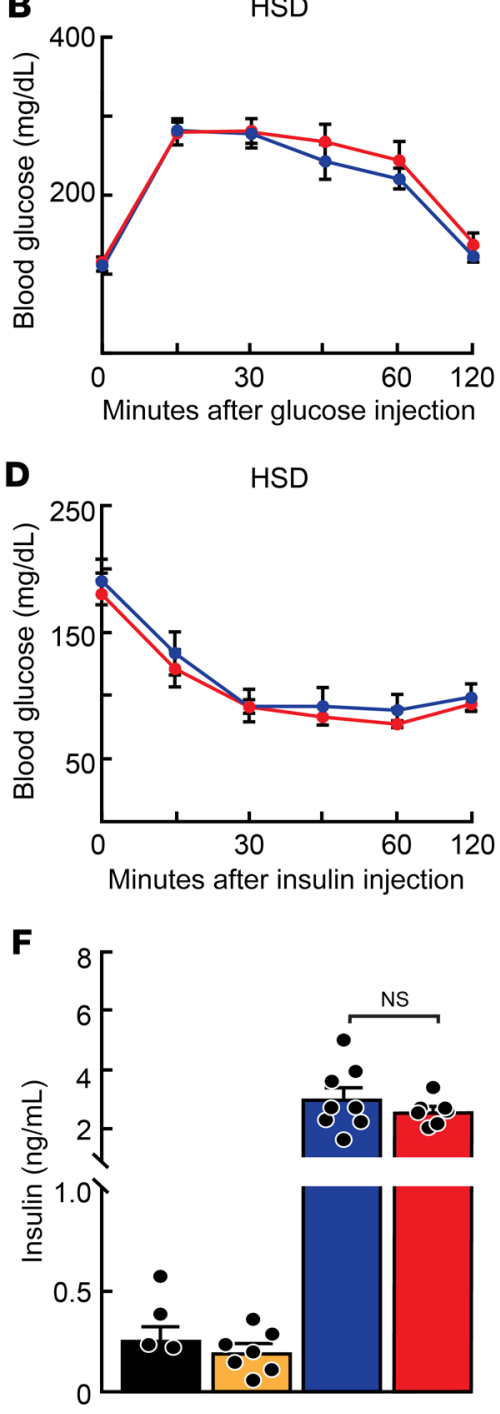

Figure 3. Hepatocyte-specific Cnr1 deletion does not affect glucose tolerance or insulin sensitivity in mice fed chow or HSD. Mice used are those described in Figure 1. Glucose and insulin tolerance tests were carried out 2 and 3 weeks prior to euthanasia, respectively. (A and $\mathbf{B}$ ) Blood glucose levels were measured at indicated times after glucose injection. (C and D) Blood glucose levels were measured at indicated times after insulin injection. (E and $\mathbf{F}$ ) Blood glucose and insulin levels after euthanasia at 22 weeks of age. Results shown as mean \pm SEM, assessed by ANOVA. The experiments were repeated in a separate cohort of mice with similar results.

CNR1 expression is low in human liver. To determine if CNR1 expression is induced in humans with NASH and/or fibrosis, we assessed CNR1 mRNA expression in livers from healthy controls and NAFLD/NASH patients via in situ hybridization (ISH). All control livers were histologically normal, while NAFLD/NASH human donors had varying NAS scores but all had a diagnosis of grade 2 fibrosis (Supplemental Table 1). In addition to the human-specific CNR1 probe, 2 control probes were included in ISH: a positive control probe for species-specific housekeeping gene PPIB to verify mRNA quality of tissue sections and a negative control probe for $D a p B$ to detect nonspecific labeling. In accordance with published findings (47), CNR1 was highly enriched in the neurons of the cerebellum, which served as the positive control tissue for the CNR1 probe (Supplemental Figure 8). All liver samples in the study were PPIB positive and $D a p B$ negative, as illustrated in the representative liver sections (Figure 7). CNR1 mRNA was expressed at extremely low levels in normal livers and NAFLD/NASH livers with no apparent differences between groups (Figure 7). Based on criteria for ISH scoring (Supplemental Table 2), CNR1 mRNA was detected at a moderate level in endothelial cells, low levels in cholangiocytes, and barely expressed in mononuclear cells and cells located within sinusoids (Supplemental Table 3). Thus, consistent with our observations in mice, CNR1 mRNA expression was low in healthy human liver samples and remain unchanged with the development of NASH.

\section{Discussion}

The endocannabinoid system, and particularly signaling through CB-1 in the liver, has been previously implicated as critical for the development of insulin resistance and NAFLD (25-29). To further investigate the possible role for CB-1 in the development and progression of NAFLD and specifically in their ability to regulate lipogenesis, we independently generated mice that lack $\mathrm{CB}-1$ in hepatocytes or HSCs. In contrast with previously published studies, we did not find that the deletion of CB-1 in hepatocytes altered lipogenesis, nor did it protect mice from the development of hepatic steatosis when fed the HSD or HFD. In addition, no changes were measured in insulin or glucose sensitivity in hepatocyte-specific-knockout mice fed chow, HSD, or HFD. Similarly, development of metabolic alterations associated with HFDs. 


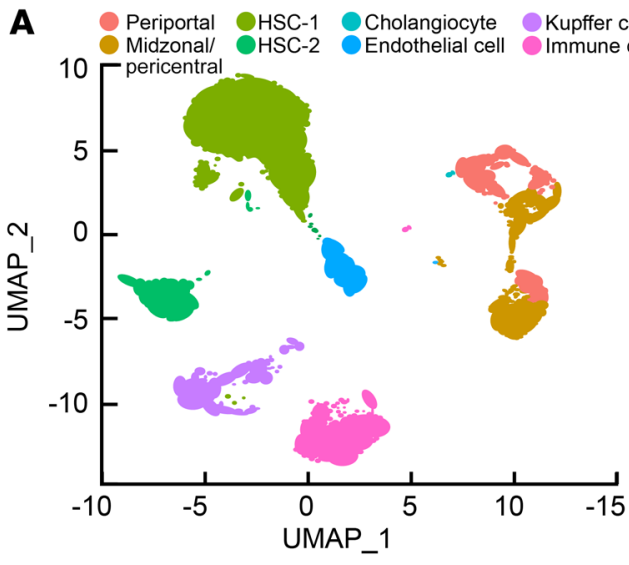

C

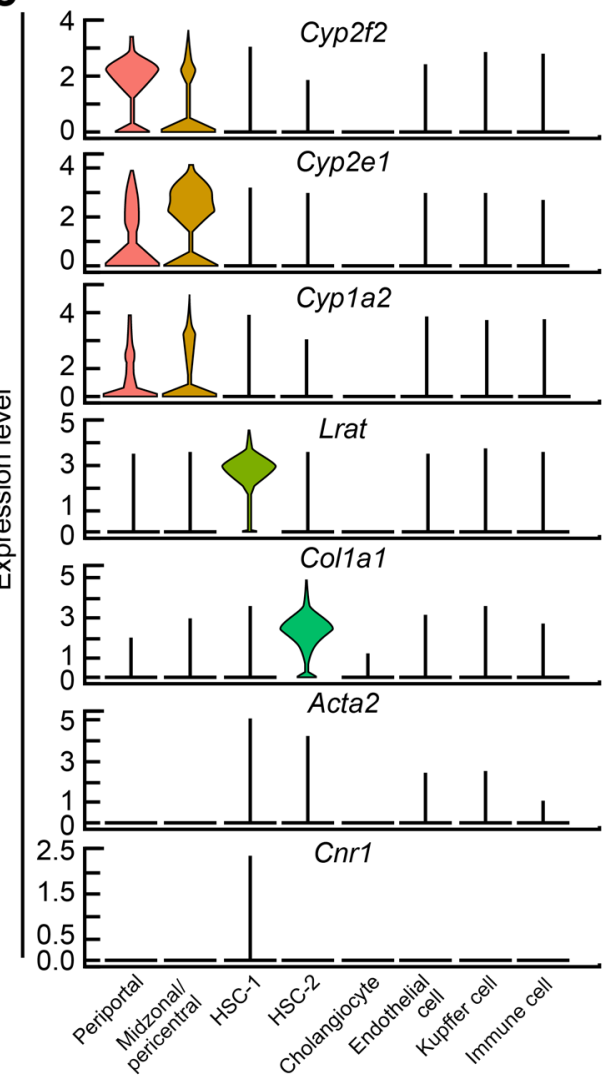

B

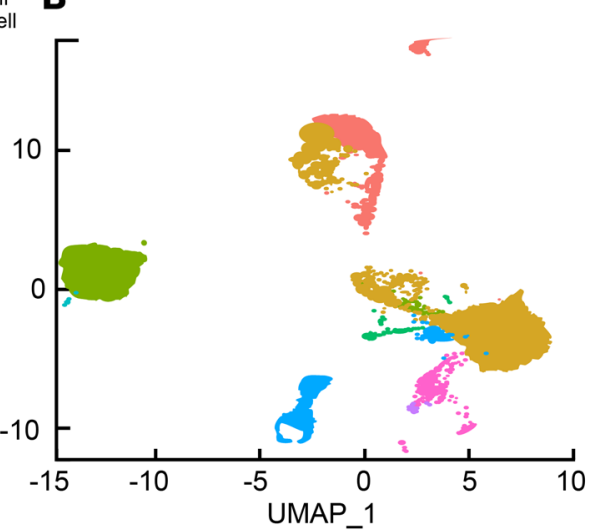

D

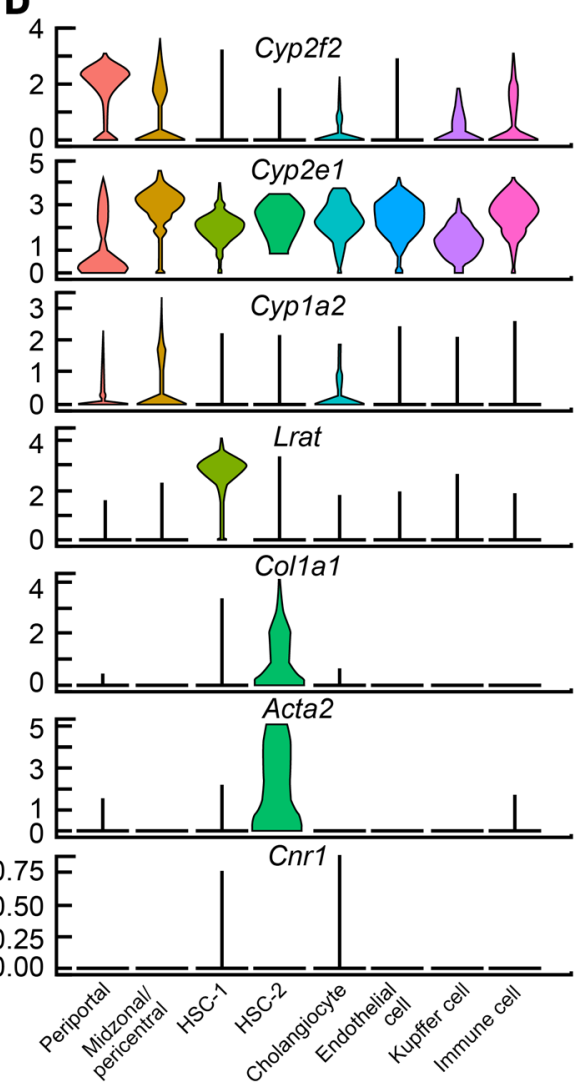

Figure 4. Single-cell RNA sequencing reveals very low $C_{n r 1}$ expression in hepatocytes and nonparenchymal liver cells of mice fed chow or HSD. Symbol UMAP plot highlighting 6 subclusters of main liver cell compartments from scRNA-seq data aggregated from chow-fed wild-type mice (A) and wild-type mice maintained on HSD for 17 weeks (B). Violin plots of representative hepatocyte zonespecific gene expression from (C) chow-fed wild-type mice and (D) wild-type mice maintained on HSD for 17 weeks: Cyp2f2 (periportal), Cyp2e1 and Cyp1a2 (midzonal/ pericentral). Violin plots of HSC landmark genes (Lrat, Col1a1, and Acta2) and Cnr1 expression across cell subpopulations from (C) chow-fed wild-type mice and (D) wildtype mice maintained on HSD for 17 weeks. the deletion of CB-1 in HSCs did not alter the development of NAFLD in HFD-fed mice, nor did it prevent the development of cirrhosis in mice administered $\mathrm{CCl}_{4}$.

As suggested in previous studies, we found the mRNA levels of Cnr1 to be extremely low in whole-liver RNA extracts and they remained low in livers of wild-type mice fed the HSD and HFD. In order to verify that our construct disrupted CB-1 expression, we bred $C n r 1^{f l / f l}$ mice with mice that express Cre under the control of the steroidogenic factor 1 (SF-1) promoter. SF-1 and CB-1 are highly expressed in the VMH (32); Cnr1 mRNA was absent in the VMH of our $C n r 1^{f l / f l} \mathrm{SF}-1-C r e$ mice. As a final attempt to determine if there was an isolated population of cells in the liver that might more highly express CB-1, we performed scRNA-seq on livers of mice fed chow or the HSD. As shown in Figure 4, low levels of Cnr1 expression were only found in HSCs and cholangiocytes.
Previous reports suggested that the pharmacological inhibition of CB-1 with SR141716A or germline deletion of Cnr1 prevented the progression of fibrosis in mouse models of liver injury, including $\mathrm{CCl}_{4}$ (44). Similarly, rimonabant was reported to reduce fibrosis in rats with $\mathrm{CCl}_{4}$-induced cirrhosis (48). These results stimulated us to further investigate the expression and function of CB-1 in HSCs. Cnr1 mRNA was expressed in HSCs isolated from wild-type mice but the expression was only slightly higher than that found in whole-liver RNA extracts by qPCR, and it was very low compared with other HSC-specific genes (Figure 5D). A greater role for CB-1 could be hypothesized if its expression were induced during HSC activation; therefore, we measured Cnr1 expression by qPCR in HSCs before and after activation in culture. While the expression of genes such as Col1a1 and $\alpha S M A$ was induced in the cultured HSCs during activation, 
A
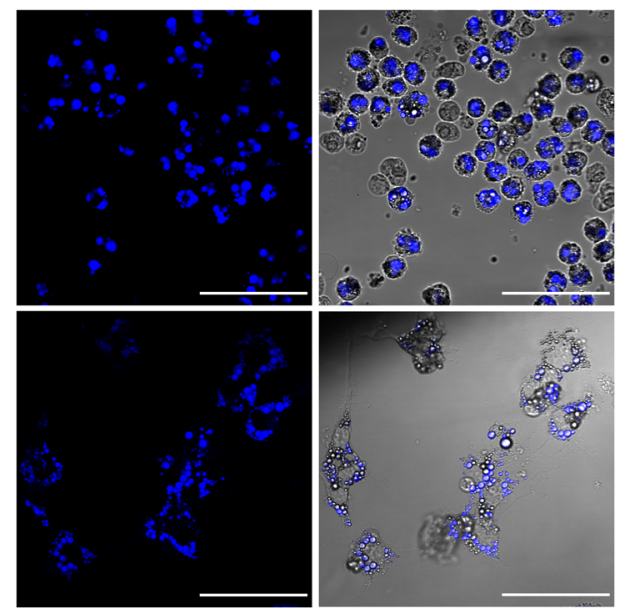

B

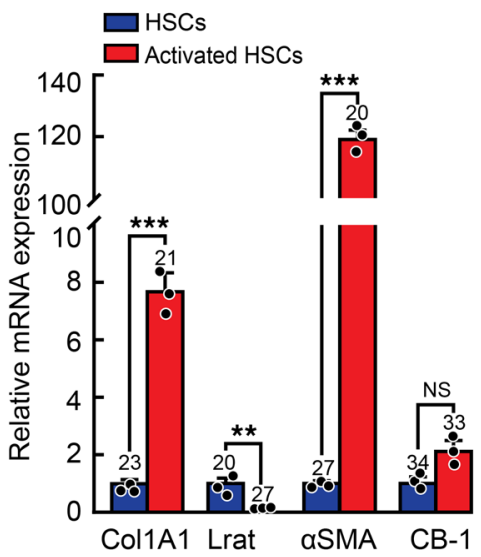

Figure 5. Cnr1 expression is low in cultured HSCs and Cnr1 deletion in HSCs does not alter $\mathrm{CCl}_{4}$-induced fibrosis. (A) Representative images of freshly isolated HSCs (upper panels) from chow-fed wild-type mice and from isolated HSCs cultured for 6 days (lower panels). Confocal microscopy was performed for detection of retinoid fluorescence (blue). Endogenous retinoid expression was visualized in cytoplasmic lipid droplets of HSCs (left panels). The merged right panels show that the retinoid signal overlaps with lipid droplets in activated HSCs. Scale bar: $50 \mu \mathrm{m}$. (B) Total RNA from freshly isolated HSCs and HSCs cultured for 6 days was extracted for qPCR quantification of Col1a1, Lrat, Acta2 ( $\alpha$ SMA), and $C n r 1$. Actb ( $\beta$-actin) was used as an invariant control. Values were expressed relative to that of freshly isolated HSCs, which was arbitrarily set to 1 . Corresponding mean $C_{t}$ values are denoted above. (C) Plasma ALT and AST levels in chow-fed doxycycline-treated $\mathrm{Hsc}-\mathrm{Cnr}^{-/-}$and $\mathrm{Cnr}^{\mathrm{fl} / \mathrm{fl}}$ mice injected with either $\mathrm{CCl}_{4}$ or corn oil (5-11/group). (D) Mice described in C were euthanized at 16 weeks of age. Total RNA was extracted from HSCs of each mouse, and the relative mRNA levels of Col1a1, Lrat, Acta2 ( $\alpha \mathrm{SMA})$, and Cnr1 were quantified by qPCR. Actb was used as an invariant control. Values were expressed relative to that of chow-fed doxycycline-treated $\mathrm{Cnr}^{f \mid l / f l}$ mice injected with corn oil, which was arbitrarily set to 1 . Corresponding mean $C$ values are denoted above. Results shown as mean \pm SEM. ${ }^{* *} P<0.01,{ }^{* *} P<0.001$ by ANOVA. (E) Gross appearance of representative livers of chow-fed, doxycycline-treated

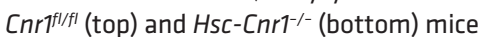
injected with $\mathrm{CCl}_{4}$ for 10 weeks. (F) $\mathrm{H} \& \mathrm{E}$ and trichrome staining of liver from mice described in E. Scale bar: $200 \mu \mathrm{m}$. All experiments (A-F) were repeated with a separate cohort of mice and the results were similar.

there was no change in expression of Cnrl (Figure 5B). In vivo studies demonstrated that the development of fibrosis induced by $\mathrm{CCl}_{4}$ was not altered in $\mathrm{Hsc}-\mathrm{Cnr1}^{-/-}$mice (Figure 5 and Supplemental Figure 7). Based on these results, we conclude that $\mathrm{CB}-1$ signaling in HSCs does not significantly reduce or augment the deposition of collagen or the development of cirrhosis in these models of hepatocyte injury.

While the studies described herein did not suggest that CB-1 is induced by HSD and HFD or that CB-1 in hepatocytes or HSCs plays a role in the development of NAFLD, it was possible that human livers have higher CB-1 expression and/or that the development of NASH further enhances CB-1 expression. ISH was used to evaluate the relative expression levels of $C n r 1$ in normal and livers with NASH (Figure 7). The results obtained from the ISH were consistent with those in the mouse studies. Cnrl mRNA levels were very low in hepatocytes and there was no discernable increase in Cnr1 expression in livers with histological evidence of NASH (Figure 7).

In summary, our studies suggest that CB-1 signaling in hepatocytes or in HSCs does not alter the development or progression of NAFLD in response to HSD or HFD in mice. There is no obvious explanation for the differences in results obtained from the studies described here and those previously published regarding the hepatocyte-specific Cnr1-knockout studies. The construct to generate $\mathrm{Cnr}^{f l / f l}$ mice contains the entire open reading frame of the Cnr1 gene (32), which is the same as that used previously (49). Moreover, the mouse strain, diet, and duration of diet feeding were identical to those used in previous publications and thus cannot be the cause of the discrepancy. One factor that cannot be controlled for that would be certainly different between centers performing the studies is the microbiome of the mice studied. The microbiome has been postulated to play a role in the development of NAFLD (50). However, if the microbiome is responsible for the differences found in this study compared with others, it would suggest that a specific microbiome is required for $\mathrm{CB}-1$ inhibition to positively impact insulin resistance and NAFLD. Such a requirement would call into question whether this therapeutic approach would have consistent and broad-based positive responses in the general population. 
A

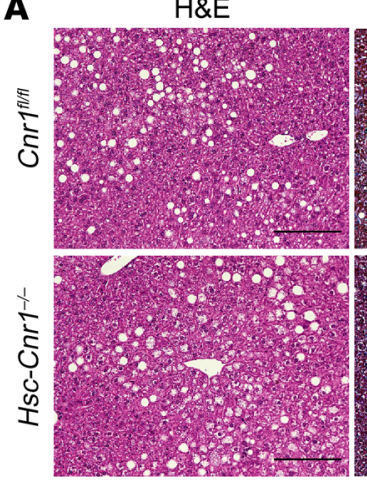

D

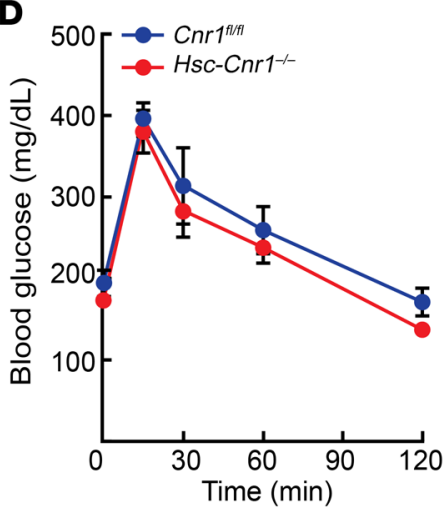

Trichrome

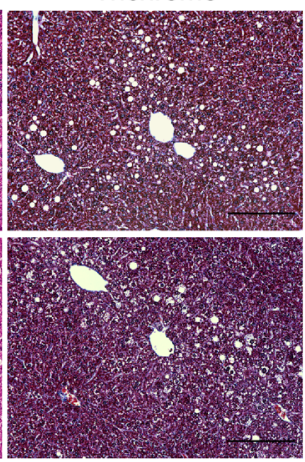

PSR

E

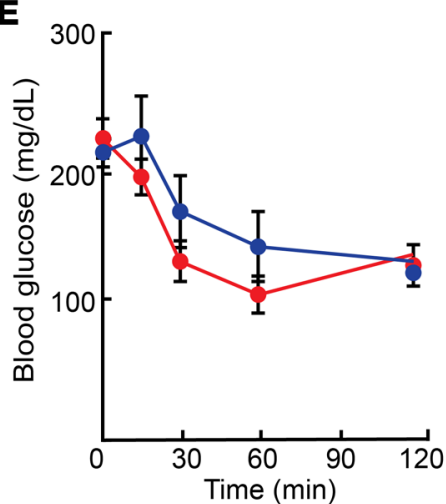

B

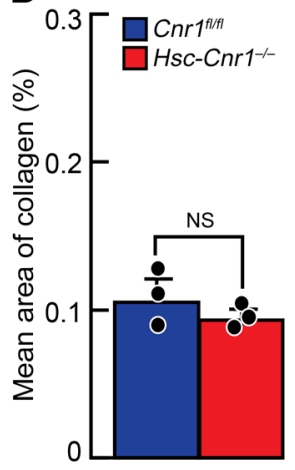

c

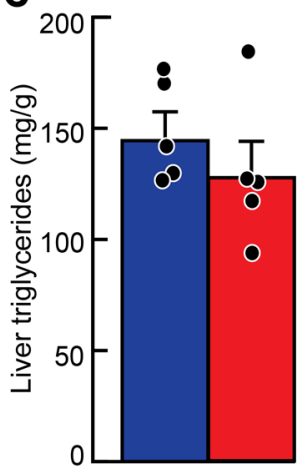

$\mathbf{F}$

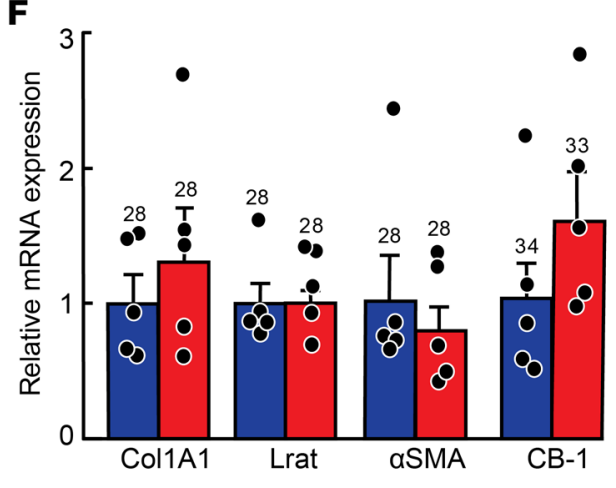

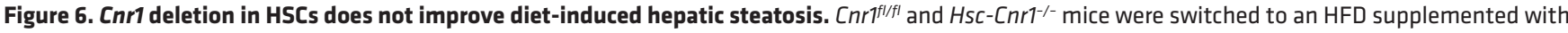
doxycycline for 12 weeks, starting at 6 weeks of age. Clucose and insulin tolerance tests were carried out 10 and 11 weeks after HFD feeding, respectively (6-7/group). (A) Representative H\&E, trichrome, and PSR staining of liver sections. Scale bar: $300 \mu \mathrm{m}$. (B) Mean area of collagen was obtained by calculating

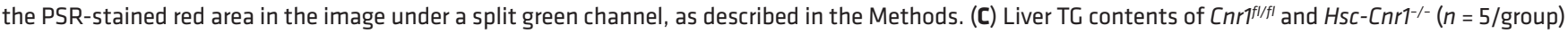
mice after 12 weeks of HFD. ( $\mathbf{D}$ and $\mathbf{E}$ ) Blood glucose levels were measured in mice at indicated times after glucose administration (D) or insulin injection (E). Mice in $\mathbf{D}$ and $\mathbf{E}$ were euthanized at 18 weeks of age. (F) Total RNA was extracted from each mouse liver, and the relative mRNA levels of Col1A1, Lrat, Acta2 ( $\alpha S M A)$, and Cnr1 were quantified by qPCR. Actb ( $\beta$-actin) was used as an invariant control. Values were expressed relative to HFD-fed, doxycycline-treated Cnr $7^{f / f l}$ mice, which was arbitrarily set to 1 . Corresponding mean $C_{t}$ values are denoted above. Results shown as mean \pm SEM, assessed by ANOVA.

Previous studies suggested that peripheral blockade of CB-1 signaling would be beneficial for the treatment of NAFLD. In fact, nimacimab, a peripherally acting antagonistic antibody against CB-1 used for the treatment of NASH, has completed a phase Ib study. Unfortunately, the results of our animal studies do not suggest that a peripherally acting compound will be successful in treating NAFLD if its mechanism of action is through hepatocytes or HSCs. While our results do not rule out the possibility that a peripherally acting CB-1 antagonist could function through other cell types and ultimately alter whole-body metabolism in a way that may improve NAFLD, it is also possible that the benefit derived is centrally mediated, which would potentially carry the same unwanted side effects associated with rimonabant.

\section{Methods}

Animals. Cnr $1^{f / f l}$ mice (C57BL/6N background) harboring the conditional floxed Cnr1 alleles were generated using CRISPR/Cas9 at the Transgenic Core Facility of the University of Texas Southwestern Medical Center.

The gRNAs and donor single-stranded oligodeoxynucleotides (ssODNs) were designed to insert one loxP site upstream and another loxP site downstream of exon 2 (Supplemental Figure 1). The
gRNA sequences are $5^{\prime}$-TCTGGGTGAGGAGACATGCCTGG-3' (upstream) and 5'-AGTCTATCGCTGCAGTTGCTCGG-3' (downstream). gRNAs were selected using the CRISPR Design Tool (http:// tools.genome-engineering.org). Cas 9 mRNA, crRNA/tracrRNA, and donor ssODNs (Sigma-Aldrich) were mixed and injected into the cytoplasm of fertilized eggs, which were then transferred into the uterus of pseudopregnant females to produced FO founders. Founders containing the dual insertion of the loxP sites were identified via PCR using primer set 5'-AGATTAGCACAGAGGCTTAT-3' and 5'-ATAAGCCTCTGTGCTAATCT-3' for the upstream loxP site, and primer set $5^{\prime}$-TCTGGGTGAGGAGACATGCC-3' and 5'-GGCATGTCTCCTCACCCAGA-3' for the downstream loxP site. FO founders were bred with C57BL/6J mice to obtain F1 mice heterozygous for the floxed $\mathrm{Cnr1}$ allele $\left(\mathrm{Cnrl}^{+/ f l}\right)$, which were then intercrossed to produce homozygous $\mathrm{Cnr}^{f l / f l}$ mice. For hepatocyte-specific deletion of Cnr1, $C n r 1^{f / f l}$ mice were bred with mice expressing Cre recombinase under the control of the albumin promotor (Alb-Cre; Jackson Laboratory, 003574). To confirm that Cnr1 was deleted in hepatocytes, liver- and tail-derived DNA was used for genotyping by PCR with the following primers: 5'-ACCACCTTCCTCATGTTAACCT-3', 5'-GACCAGAGACAGCTCCAGA-3', and 5'-TGAGGGCTATATTCTGTTTTTGC-3' (wild type, $195 \mathrm{bp}$; flox, $233 \mathrm{bp}$; delta, $480 \mathrm{bp}$ ). 


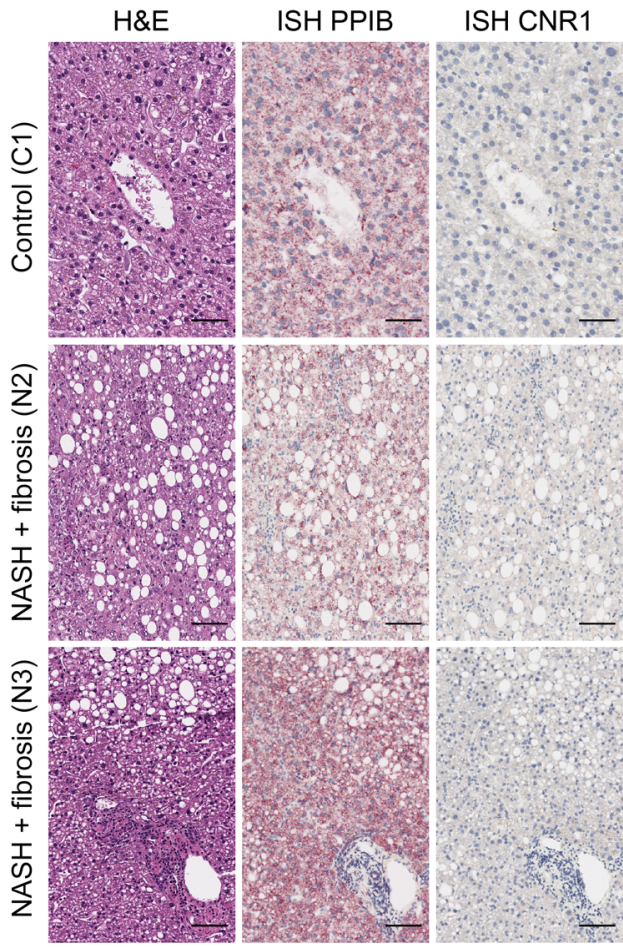

Figure 7. CNR1 mRNA expression in control and NASH human livers. Left panel: Representative H\&E staining of livers from normal individuals $(n=$ 3 ) or patients with NASH $(n=7)$. Middle panel: Representative ISH staining of the PPIB housekeeping gene showing the detection of red punctate dots in hepatocytes of normal $(n=3)$ and NASH livers $(n=7)$. Right panel: Representative ISH revealing a negative CRN1 mRNA expression in hepatocytes of normal $(n=3)$ and NASH livers $(n=7)$. Scale bar: $50 \mu \mathrm{m}$.

To achieve inducible HSC Cnr1 gene deletion, $C n r^{1 / f l}$ mice were bred with TRE-Cre mice (Jackson Laboratory, 006234). Offspring with both targeted alleles and the Cre transgene were bred with mice expressing rtTA driven by the Lrat promoter. $C n r 1^{f / f l}$ mice with Cre and rtTA were fed a doxycycline diet (BioServ, S3888) for 4 weeks to obtain $\mathrm{Hsc}$-Cnr1-/- mice.

Diet-induced hepatic steatosis. Mice were housed at room temperature $\left(23^{\circ} \mathrm{C}\right)$ and maintained on a 12-hour light/12-hour dark cycle and provided ad libitum access to rodent chow (Harlan, Teklad Global 18\% Protein Rodent Diet 2018; 18\% kcal from fat, $3.1 \mathrm{kcal} / \mathrm{g})$, HSD (17\% kcal from sucrose, $45 \%$ kcal from fat; Research Diets, D12451), or HFD (60\% kcal from fat; Research Diets, D12492). Body weights were measured weekly and mice in the HSD and HFD studies were euthanized at 22 and 16 weeks of age, respectively. For diet-induced obesity studies in HSC-specific Cnr1-knockout mice, $\mathrm{Hsc}^{-\mathrm{Cnr1}^{-/-}}$and $\mathrm{Cnr1}^{\mathrm{fl/fl}}$ mice were fed the HFD supplemented with $600 \mathrm{mg} / \mathrm{kg}$ doxycycline (60\% kcal from fat; BioServ) for 12 weeks starting at 4 weeks of age.

$\mathrm{CCl}_{4}$-induced liver fibrosis. $\mathrm{Hsc}-\mathrm{Cnrl}^{-/-}$and $\mathrm{Cnr}^{\mathrm{fl/l} / \mathrm{l}}$ mice were fed the rodent chow described above supplemented with $600 \mathrm{mg} / \mathrm{kg}$ doxycycline (BioServ, S3888) for 4 weeks to induce Cnr1 deletion in HSCs. Mice were injected intraperitoneally with either $\mathrm{CCl}_{4}(0.2 \mathrm{~mL} /$ $\mathrm{kg}$ of body weight; Sigma-Aldrich, 289116) or corn oil (Sigma-Aldrich, C8267) twice a week for 10 weeks. Mice were maintained on doxycycline diet, and body weights were measured weekly after injection and euthanized at 16 weeks of age.
HSC isolation. HSCs were isolated and cultured as described previously (46). RNA was isolated from HSCs for reverse transcription and quantitative real-time PCR analysis, as described previously (51).

Glucose tolerance and insulin sensitivity studies. To determine glucose tolerance, 16-hour-fasted mice were administered glucose intraperitoneally ( $1.5 \mathrm{~g} / \mathrm{kg}$ of body weight for chow-fed and HSD-fed mice). Tail vein blood was collected at $0,15,30,60$, and 120 minutes after glucose injection. To determine insulin sensitivity, 6-hour-fasted mice were administered insulin intraperitoneally $(1 \mathrm{U} / \mathrm{kg}$ and $2 \mathrm{U} / \mathrm{kg}$ of body weight for chow-fed and HSD-fed mice, respectively; Humulin $\mathrm{R}$, Lilly). Tail vein blood was collected at $0,15,30,60$, and 120 minutes after insulin injection. Blood glucose levels were quantified using a Bayer Contour glucose meter.

HFD-fed, doxycycline-inducible $\mathrm{Hsc}_{-} \mathrm{Cnrl}^{-/-}$and control mice $\left(\mathrm{Cnr} \mathrm{1}^{\mathrm{fl} / \mathrm{fl}}\right)$ were administered glucose $(2 \mathrm{~g} / \mathrm{kg}$ of body weight; SigmaAldrich) via oral gavage or human recombinant insulin $(0.75 \mathrm{U} / \mathrm{kg}$ of body weight; Novo Nordisk) via intraperitoneal injection, respectively. Blood glucose levels were determined with a glucometer at indicated time points.

Liver function tests. Plasma ALT, plasma AST, and liver TG and cholesterol levels were measured as previously described (51).

Histology and collagen quantification. All histological sections, hematoxylin \& eosin (H\&E), trichrome, and PSR staining were performed by the Molecular Pathology Core at the University of Texas Southwestern Medical Center. The mean area of PSR-stained collagen in each liver section was determined using standard ImageJ software (NIH). PSR-stained liver sections display red collagen under light microscopy (brightfield). The images were separated into 3 distinct channels. Collagen in liver sections of $\mathrm{CCl}_{4}$-injected mice were quantified by image reduction to the green channel followed by automatic calculation of the mean red area (minimum $=0$, maximum $=255$ ). To better reflect the whole tissue, each PSR-stained liver slide was subdivided into 5 to 10 small sections for individual quantification, and the average was obtained for plotting.

RNA isolation and quantitative real-time PCR. After total RNA extraction from tissues and cells, RT-PCR and quantitative realtime PCR were performed as previously described (51). All data were normalized to the expression of mouse $\beta$-actin or apolipoprotein $\mathrm{B}$. The following forward and reverse primers were used for real-time PCR: Cnr1, 5'-GTACCATCACCACAGACCTCCT-3' and 5'-GGATTCAGAATCATGAAGCACTCCA-3'; ApoB, 5'-CGTGGGCTCCAGCATTCTA-3' and 5'-TCACCAGTCATTTCTGCCTTTG-3'; Col1a1, 5'-CGTCTGGTTTGGAGAGAGCAT-3' and 5'-GGTCAGCTGGATAGCGACATC-3'; Lrat, 5'-ACACTGACATCATTCTACTCTCTTTGG-3' and 5'-AGTTCAATGATTCTGGTGTTGTGTAAC-3'; $\alpha S M A, 5^{\prime}$-TTCCGCTGCCCAGAGACT-3' and 5'-GATGCCCGCTGACTCCAT-3'; Srebp-1c, 5'-GGAGCCATGGATTGCACATT-3' and 5'-GGCCCGGGAAGTCACTGT-3'; Srebp-2, 5'-GCGTTCTGGAGACCATGGA-3' and 5'-ACAAAGTTGCTCTGAAAACAAATCA-3'; Chrebp, 5'-CCTTCGCCAACTCAGCACTT-3' and $5^{\prime}$-TGGCTTGCTCAGGCACAA-3'; Acly, 5'-GCCAGCGGGAGCACATC-3' and 5'-CTTTGCAGGTGCCACTTCATC-3'; Acc1, $5^{\prime}$-TGGACAGACTGATCGCAGAGAAAG- $3^{\prime}$ and $5^{\prime}$-TGGAGAGCCCCACACACA-3'; Fasn, 5'-GCTGCGGAAACTTCAGGAAAT-3' and $5{ }^{\prime}$-AGAGACGTGTCACTCCTGGACTT-3'; Scd1, 5' ${ }^{\prime}$-CCGGAGACCCCTTAGATCGA-3' and 5'-TAGCCTGTAAAAGATTTCTGCAAACC-3'; Elovl6, 5'-TGTACGCTGCCTTTATCTTTGG-3' and 
5'-GCGGCTTCCGAAGTTCAA-3'; TRE-Cre, 5'-GATTTCGACCAGGTTCGTTC-3' and 5'-GCTAACCAGCGTTTTCGTTC-3'.

Immunoblots. Liver membrane and nuclear proteins were isolated from liver as previously described (51). Equal aliquots of liver membrane or nuclear proteins from 7 to 10 mice per group were pooled and $50 \mu \mathrm{g}$ of protein was separated by SDS-PAGE and immunoblot analyses were carried out as previously described (51). SREBP-1 was detected using a rabbit polyclonal anti-SREBP-1 (IgG-20B12) antibody and SREBP-2 was detected using a rabbit monoclonal anti-SREBP-2 (IgG-22D5) antibody, both generated in house $(52,53)$. Calnexin was used as an invariant control for membrane protein and was detected using a commercially available polyclonal antibody (Enzo Life Science, lot ADI-SPA-860-F). Creb (cAMP response element-binding protein) was used as an invariant control protein for nuclear protein immunoblots and was detected using a commercially available monoclonal antibody (Invitrogen, catalog 35-0900). Bound anti-SREBP-1, anti-SREBP-2, and anti-calnexin primary antibodies were visualized using peroxidase-conjugated, affinity-purified, light-chain specific, goat anti-rabbit IgG secondary antibody (Jackson ImmunoResearch Laboratories Inc., 111-035-047). Bound anti-Creb antibody was visualized using peroxidase-conjugated, affinity-purified, light-chain specific, goat anti-mouse IgG secondary antibody (Jackson ImmunoResearch Laboratories Inc., 115-035-072).

Immunofluorescence microscopy of HSCs. Freshly isolated HSCs were resuspended in phenol red-free DMEM containing 10\% FBS, 5\% streptomycin, and $1 \%$ HEPES, and cultured in glass-bottom dishes at $37^{\circ} \mathrm{C}$ under $5 \% \mathrm{CO}_{2}$. HSCs cultured for 1 day and 6 days were visualized via confocal microscopy (Zeiss LSM 880). Endogenous retinoid fluorescence signal was detected with the laser set up for detection of DAPI.

ISH. ISH was performed using the RNAscope 2.5 LS Reagent KitRed (catalog 322150) from Advanced Cell Diagnostics according to the manufacturer's guidelines on a Leica Bond Rx (Leica Biosystems). The RNAscope LS 2.5 Hs-CNR1 probe (catalog 591528) was used to identify human CNR1 mRNA in formalin-fixed, paraffin-embedded sections of human cerebellum and liver samples from healthy and diseased donors. Tissues were sectioned at $5 \mu \mathrm{m}$ on Fisherbrand Superfrost Plus microscope slides (Thermo Fisher Scientific, catalog 12-550-15). The mRNA quality of tissue sections was verified by probing for the housekeeping gene PPIB using RNAscope 2.5 LS Positive Control Probe Hs-PPIB (catalog 313908), and nonspecific labeling was tested by using RNAscope 2.5 LS Negative Control Probe DapB (catalog 312038). Tissue sections were pretreated using the following conditions: 15 minutes ER2 and 15 minutes protease. Following automation, slides were individually dipped into fresh xylene and coverslipped using VectaMount Permanent Mounting Medium (Vector Labs, catalog H-5000).

scRNA-seq. Two pairs of mice (one pair fed chow, one pair fed the HSD) were used for scRNA-seq. For each pair, one mouse was perfused for hepatocyte preparation, and the other for HSC preparation. HSC isolation was as described previously (46). Hepatocytes were isolated using pronase/collagenase digestion. After Nycodenz gradient centrifugation, the entire supernatant was removed and the pellet was suspended with $1 \mathrm{~mL}$ chilled PBS in a 1.5-mL Eppendorf tube. The cell suspension was centrifuged at $800 \mathrm{rpm}$ for 3 minutes. The superna- tant was discarded and the pellet was washed with $1 \mathrm{~mL}$ chilled PBS. Hepatocytes were resuspended in chilled PBS for cell counting. The approximate cell viability was between $80 \%$ and $90 \%$, as determined by trypan blue staining. The final single-cell suspension was used to generate separate libraries for hepatocytes and HSCs via the 10x Genomics Chromium Single Cell 3' Library and Gel Beads Kit (version 3) according to the manufacturer's protocol. Libraries were sequenced on an Illumina NextSeq 500 with high output of 400 million reads.

Raw data were demultiplexed using Cell Ranger (version 3.1.0) and barcode-aware wrapper (version 2.17.1.14) supported by the $10 \times$ Genomics pipeline. Transcriptomes were aligned to Genome Reference Consortium Mouse Build 38 ( $\mathrm{mm} 10$ ) using STAR. Approximately $68.5 \%$ of sequencing reads were confidently mapped to the mouse genome. Seurat (version 3.2.0) was applied for single-cell transcriptome analysis and plots in $\mathrm{R}$ (version 3.5.1). Briefly, genes that were expressed in over 3 cells were kept as well as cells expressing more than 200 unique genes. Multiplets were excluded by removing cells with greater than 3,000 unique genes. Data were scaled and mitochondrial genes were removed for deep analysis. Then, UMI counts were scaled and variations due to differences in UMI/cell and percentage mitochondrial genes were regressed out of the data. Cells in each pair of mice fed chow or HSD were integrated to better understand cell identity and function (37). The genes with the greatest variability were used for UMAP visualization and clustering. Subclustering of hepatocytes and HSCs was based on different resolutions. All scRNA-seq data were deposited into NCBI's Gene Expression Omnibus database (GEO GSE182365).

Statistics. Results are expressed as mean \pm SEM. Comparisons were performed with 2-tailed, unpaired Student's $t$ test or 1-way ANOVA followed by post hoc comparison with Tukey's correction using GraphPad Prism 7 software.

Study approval. All animal studies were approved and conducted under the oversight of the University of Texas Southwestern Institutional Animal Care and Use Committee.

\section{Author contributions}

SW and QZ conducted experiments. JDH, PES, and JKE designed the research. SW and JDH wrote the manuscript. SZ and PES provided $\mathrm{Hsc}_{-} \mathrm{Cnrl}^{-/-}$founder mice. TF, MB, KKB, and ML contributed to the human CNR1 ISH study. CMC and JKE generated and verified the $\mathrm{CnrI}^{-/-}$mice. GL and JDH revised the manuscript.

\section{Acknowledgments}

We thank Norma Anderson, Judy Sanchez, Tuyet Dang, and Tessa Edwards for excellent technical assistance; Gervaise Henry and Chelsea Hepler for critical advice on scRNA-seq experiments; and Chelsea Burroughs and Nancy Heard for assistance with graphics. This work was supported by NIH grant HL-20948.

Address correspondence to: Jay D. Horton, Departments of Internal Medicine and Molecular Genetics, UT Southwestern Medical Center, 5323 Harry Hines Blvd. Dallas, Texas 75390-9046, USA. Phone: 214.648.9677; Email: Jay.Horton@UTSouthwestern.edu.
1. Chalasani N, et al. The diagnosis and management of nonalcoholic fatty liver disease: practice guidance from the American Association for the Study of Liver Diseases. Hepatology.
2018;67(1):328-357.

2. Browning JD, et al. Prevalence of hepatic steatosis in an urban population in the United States: impact of ethnicity. Hepatology.
2004;40(6):1387-1395.

3. Perumpail BJ, et al. Clinical epidemiology and disease burden of nonalcoholic fatty liver disease. World J Gastroenterol. 2017;23(47):8263-8276. 
4. Cohen JC, et al. Human fatty liver disease: old questions and new insights. Science. 2011;332(6037):1519-1523.

5. Browning JD, Horton JD. Molecular mediators of hepatic steatosis and liver injury. JClin Invest. 2004;114(2):147-152.

6. Smith GI, et al. Insulin resistance drives hepatic de novo lipogenesis in nonalcoholic fatty liver disease. JClin Invest. 2020;130 (3):1453-1460.

7. Shimomura I, et al. Increased levels of nuclear SREBP-1c associated with fatty livers in two mouse models of diabetes mellitus. J Biol Chem. 1999;274(42):30028-30032.

8. Pettinelli P, et al. Enhancement in liver SREBP-1c/ PPAR-alpha ratio and steatosis in obese patients: correlations with insulin resistance and n-3 longchain polyunsaturated fatty acid depletion. Biochim Biophys Acta. 2009;1792(11):1080-1086.

9. Uyeda K, et al. Carbohydrate responsive element-binding protein (ChREBP): a key regulator of glucose metabolism and fat storage. Biochem Pharmacol. 2002;63(12):2075-2080.

10. Linden AG, et al. Interplay between ChREBP and SREBP-1c coordinates postprandial glycolysis and lipogenesis in livers of mice. J Lipid Res. 2018;59(3):475-487.

11. Kim CW, et al. Acetyl CoA carboxylase inhibition reduces hepatic steatosis but elevates plasma triglycerides in mice and humans: a bedside to bench investigation. Cell Metab. 2017;26(2):394-406.

12. Moon YA, et al. The Scap/SREBP pathway is essential for developing diabetic fatty liver and carbohydrate-induced hypertriglyceridemia in animals. Cell Metab. 2012;15(2):240-246.

13. Gluchowski NL, et al. Hepatocyte deletion of triglyceride-synthesis enzyme acyl CoA: diacylglycerol acyltransferase 2 reduces steatosis without increasing inflammation or fibrosis in mice. Hepatology. 2019;70(6):1972-1985.

14. Loomba R, et al. GS-0976 reduces hepatic steatosis and fibrosis markers in patients with nonalcoholic fatty liver disease. Gastroenterology. 2018;155(5):1463-1473.

15. Esler WP, Bence KK. Metabolic targets in nonalcoholic fatty liver disease. Cell Mol Gastroenterol Hepatol. 2019;8(2):247-267.

16. Newsome PN, et al. A placebo-controlled trial of subcutaneous semaglutide in nonalcoholic steatohepatitis. N EnglJMed.2021;384(12):1113-1124.

17. Mantovani A, et al. Glucagon-like peptide-1 receptor agonists for treatment of nonalcoholic fatty liver disease and nonalcoholic steatohepatitis: an updated meta-analysis of randomized controlled trials. Metabolites. 2021;11(2):73.

18. Vilar-Gomez E, et al. Weight loss through lifestyle modification significantly reduces features of nonalcoholic steatohepatitis. Gastroenterology. 2015;149(2):367-378.

19. Lassailly G, et al. Bariatric surgery reduces features of nonalcoholic steatohepatitis in morbidly obese patients. Gastroenterology. 2015;149(2):379-388.

20. Jesudason D, Wittert G. Endocannabinoid system in food intake and metabolic regulation. Curr Opin Lipidol. 2008;19(4):344-348.

21. Cristino L, et al. Cannabinoids and the expanded endocannabinoid system in neurological disorders. Nat Rev Neurol. 2020;16(1):9-29.

22. Christopoulou FD, Kiortsis DN. An overview of the metabolic effects of rimonabant in randomized controlled trials: potential for other cannabinoid 1 receptor blockers in obesity. J Clin Pharm Ther. 2011;36(1):10-18.

23. Despres JP, et al. Effects of rimonabant on metabolic risk factors in overweight patients with dyslipidemia. N Engl J Med.2005;353(20):2121-2134

24. Akbas F, et al. A critical review of the cannabinoid receptor as a drug target for obesity management. Obes Rev. 2009;10(1):58-67.

25. Tam J, et al. Endocannabinoids in liver disease. Hepatology. 2011;53(1):346-355.

26. Osei-Hyiaman D, et al. Endocannabinoid activation at hepatic $\mathrm{CB} 1$ receptors stimulates fatty acid synthesis and contributes to diet-induced obesity. JClin Invest. 2005;115(5):1298-1305.

27. Osei-Hyiaman D, et al. Hepatic CB1 receptor is required for development of diet-induced steatosis, dyslipidemia, and insulin and leptin resistance in mice. J Clin Invest. 2008;118(9):3160-3169.

28. Liu J, et al. Hepatic cannabinoid receptor-1 mediates diet-induced insulin resistance via inhibition of insulin signaling and clearance in mice. Gastroenterology. 2012;142(5):1218-1228.

29. Liu J, et al. Cannabinoid-1 receptor antagonism improves glycemic control and increases energy expenditure through sirtuin $-1 /$ mechanistic target of rapamycin complex 2 and 5 'adenosine monophosphate-activated protein kinase signaling. Hepatology. 2019;69(4):1535-1548.

30. Mallat A, et al. Cannabinoid signaling and liver therapeutics. J Hepatol. 2013;59(4):891-896.

31. de Vries WN, et al. Expression of Cre recombinase in mouse oocytes: a means to study maternal effect genes. Genesis. 2000;26(2):110-112.

32. Castorena CM, et al. CB1Rs in VMH neurons regulate glucose homeostasis but not body weight. Am Physiol Endocrinol Metab. 2021;321(1):E146-E155.

33. Quarta $\mathrm{C}$, et al. $\mathrm{CB}(1)$ signaling in forebrain and sympathetic neurons is a key determinant of endocannabinoid actions on energy balance. Cell Metab. 2010;11(4):273-285.

34. Dhillon $\mathrm{H}$, et al. Leptin directly activates SF1 neurons in the $\mathrm{VMH}$, and this action by leptin is required for normal body-weight homeostasis. Neuron. 2006;49(2):191-203.

35. MacParland SA, et al. Single cell RNA sequencing of human liver reveals distinct intrahepatic macrophage populations. Nat Commun.
2018;9(1):4383

36. Butler A, et al. Integrating single-cell transcriptomic data across different conditions, technologies, and species. Nat Biotechnol.2018;36(5):411-420.

37. Stuart T, et al. Comprehensive integration of single-cell data. Cell. 2019;177(7):1888-1902.

38. Becht $\mathrm{E}$, et al. Dimensionality reduction for visualizing single-cell data using UMAP. Nat Biotechnol. 2019;37(1):38-44

39. Halpern KB, et al. Single-cell spatial reconstruction reveals global division of labour in the mammalian liver. Nature. 2017;542(7641):352-256.

40. Droin C, et al. Space-time logic of liver gene expression at sub-lobular scale. Nat Metab. 2021;3(1):43-58.

41. Aizarani N, et al. A human liver cell atlas reveals heterogeneity and epithelial progenitors. Nature. 2019;572(7768):199-204.

42. Kietzmann T. Metabolic zonation of the liver: the oxygen gradient revisited. Redox Biol. 2017;11:622-630

43. Tsuchida T, Friedman SL. Mechanisms of hepatic stellate cell activation. Nat Rev Gastroenterol Hepatol. 2017;14(7):397-411.

44. Teixeira-Clerc F, et al. CB1 cannabinoid receptor antagonism: a new strategy for the treatment of liver fibrosis. Nat Med. 2006;12(6):671-676

45. Mederacke I, et al. Fate tracing reveals hepatic stellate cells as dominant contributors to liver fibrosis independent of its aetiology. Nat Commun. 2013;4:2823.

46. Mederacke I, et al. High-yield and high-purity isolation of hepatic stellate cells from normal and fibrotic mouse livers. Nat Protoc. 2015;10(2):305-315.

47. Ashton JC, et al. Immunohistochemical localization of cannabinoid CB1 receptor in inhibitory interneurons in the cerebellum. Cerebellum. 2004;3(4):222-226

48. Giannone FA, et al. Reversal of liver fibrosis by the antagonism of endocannabinoid CB1 receptor in a rat model of $\mathrm{CCl}(4)$-induced advanced cirrhosis. Lab Invest. 2012;92(3):384-395.

49. Marsicano G, et al. The endogenous cannabinoid system controls extinction of aversive memories. Nature. 2002;418(6897):530-534.

50. Quesada-Vazquez S, et al. Diet, gut microbiota and non-alcoholic fatty liver disease: three parts of the same axis. Cells. 2020;9(1):E176.

51. Rong S, et al. Expression of SREBP-1c requires SREBP-2-mediated generation of a sterol ligand for LXR in livers of mice. Elife. 2017;6:e25015.

52. Engelking LJ, et al. Overexpression of Insig-1 in the livers of transgenic mice inhibits SREBP processing and reduces insulin-stimulated lipogenesis. JClin Invest. 2004;113(8):1168-1175.

53. McFarlane MR, et al. Scap is required for sterol synthesis and crypt growth in intestinal mucosa. JLipid Res. 2015;56(8):1560-1571. 